\title{
Single-Session vs Multiple-Session Pattern Scanning Laser Panretinal Photocoagulation in Proliferative Diabetic Retinopathy
}

\author{
The Manchester Pascal Study
}

Mahiul M. K. Muqit, MRCOphth; George R. Marcellino, PhD; David B. Henson, PhD; Lorna B. Young, MBChB; Niall Patton, FRCOphth; Stephen J. Charles, FRCOphth; George S. Turner, FRCOphth; Paulo E. Stanga, MD

Objective: To investigate the effects of pattern scanning laser (Pascal; OptiMedica, Santa Clara, California) multispot panretinal photocoagulation given in a singlesession (SS-PRP) vs single-spot multiple-session PRP (MS-PRP) on proliferative diabetic retinopathy (PDR).

Methods: Single-center, randomized clinical trial of 40 eyes. Proliferative diabetic retinopathy was treated with a 400- $\mu \mathrm{m}$ spot size in 1500 burns given either as Pascal in 20-millisecond SS-PRP or in 3 sessions (100millisecond MS-PRP) during a 4-week period. Visual acuity, central subfield retinal thickness (CRT), and 24-2 Swedish interactive thresholding algorithm visual fields were recorded at baseline and 4 and 12 weeks.

Main Outcome Measures: Central subfield retinal thickness, mean deviation, and PDR grade at 12 weeks.

Results: There was a significant increase in mean CRT with MS-PRP (22 $\mu \mathrm{m}$ at 4 weeks, 95\% CI, -32.25 to $-10.75 ; 20 \mu \mathrm{m}$ at 12 weeks, $95 \%$ CI, -28.75 to -10.82 ; $P<.001$ ) and no significant increase in the SS-PRP group. The mean deviation increased significantly in the SSPRP group after 4 weeks $(0.73 \mathrm{~dB}, P=.048)$, with no sig- nificant changes in either group at other points. A positive effect on PDR was observed in $74 \%$ of eyes in the SS-PRP group vs 53\% in the MS-PRP group $(P=.31)$. Mean treatment time for SS-PRP was 5.04 minutes (SD, 1.5 minutes) compared with 59.3 (SD, 12.7 minutes) in the MSPRP group $(P<.001)$.

Conclusions: There were no adverse outcomes (CRT, visual acuity, or visual field) from using multispot SSPRP vs single-spot MS-PRP at 12 weeks postlaser, and treatment times were significantly shorter for multispot SS-PRP. Pascal SS-PRP was as effective as MS-PRP in the treatment of PDR.

Application to Clinical Practice: Twenty-millisecond Pascal SS-PRP may be safely and rapidly performed in 1500 burns with a similar efficacy to conventional MS-PRP.

Trial Identifier: Research and Development Office PIN R00037, Central Manchester University Hospitals Foundation Trust.

Arch Ophthalmol . 2010;128(5):525-533
Author Affiliations: Manchester Royal Eye Hospital (Drs Muqit, Henson, Young, Patton, Charles, Turner, and Stanga), and University of Manchester (Drs Muqit, Henson, and Stanga), Manchester, England; and OptiMedica Corporation, Santa Clara, California (Dr Marcellino).

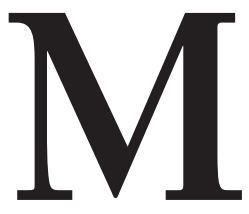

ORE THAN 20 YEARS ago, the Diabetic Retinopathy Study established conventional argon green laser panretinal photocoagulation (PRP) standards for treating proliferative diabetic retinopathy (PDR). ${ }^{1}$ The Early Treatment Diabetic Retinopathy Study (ETDRS) subsequently demonstrated that visible end point PRP remains the gold standard for first-line PDR treatment. ${ }^{2}$ More recently, clinical investigators have examined effects of short-, microsecond-, and ultrashort-pulse laser photocoagulation techniques. ${ }^{3-5}$ This is the first prospective randomized trial to validate the potential efficacy of nonconventional photocoagu- lation treatments on safety profile, disease activity, and visual outcomes in PDR.

Sight-threatening macular edema is an uncommon complication following PRP. ${ }^{6-8}$ The aim of new laser technology is to achieve retinal photocoagulation that allows for the development of healing responses by selectively targeting the retinal pigment epithelium with minimal photoreceptor loss and scar expansion, and subsequent photoreceptor and retinal pigment epithelium cell repopulation. ${ }^{9}$

Visual field loss after both full and scatter PRP has been reported by a number of studies and may be related to laser burn expansion over time. ${ }^{10-12}$ In relation to the field defect, PRP has been shown to cause a loss to the binocular field that may re- 
Table 1. Study Eye Major Inclusion and Exclusion Criteria

Inclusion Criteria

Aged $>18$ years

Patients with diabetes mellitus type I or type 2 who meet the WHO or

ADA criteria for diabetes

ETDRS visual acuity 35-85 letters (Snellen equivalent of $6 / 60$ or better)

Newly diagnosed PDR

Mean CRT $<300 \mu \mathrm{m}$ as measured by OCT scans with absence of

intraretinal and/or subretinal fluid

Adequate pupil dilatation and clear media to perform laser

photocoagulation, digital photography, and OCT

Ability to perform accurate Humphrey visual field test

Exclusion Criteria

Recent (last 6 months) or ongoing poor glycemic control;

$\mathrm{HbA}_{1 \mathrm{c}}>10.0 \mathrm{mg} / \mathrm{dL}$

Uncontrolled hypertension; blood pressure $\geq 180 / 110 \mathrm{~mm} \mathrm{Hg}$

History of chronic renal failure or renal transplant for diabetic nephropathy

Lens opacity/cataract that could influence vision and results

Any previous surgical or laser treatment to the study eye or fellow eye

Planned YAG peripheral iridotomy

Previous laser photocoagulation or macular laser treatment to the

study eye or fellow eye

History of DME in study or fellow eye

Any previous ocular condition that may be associated with a risk of

macular edema

Active eyelid or adnexal infection

Previous retinal treatment: laser, drug, or surgery

Planned intraocular surgery within 1 year

Abbreviations: ADA, American Diabetic Association; CRT, retinal thickness within central subfield; DME, diabetic macular edema; ETDRS, Early

Treatment Diabetic Retinopathy Study; $\mathrm{HbA}_{1 c}$, hemoglobin $\mathrm{A}_{1 \mathrm{c}} ; \mathrm{OCT}$, optical coherence tomography; PDR, proliferative diabetic retinopathy; WHO, World Health Organization.

sult in the patient not meeting the standard required by the United Kingdom (UK) Driving and Vehicle Licensing Authority (DVLA). This can have a major impact on a patient's quality of life, especially younger individuals with diabetes. ${ }^{13}$

In 1982, Doft and Blankenship ${ }^{14}$ investigated singlesession PRP (SS-PRP) vs multiple-session PRP (MSPRP) and did not report long-term increased complications in either group. Although significant complications such as choroidal and retinal detachment occurred postlaser, these events were transient. Significant visual field loss was again observed in $44 \%$ to $48 \%$ of patients.

A recent study by the Diabetic Retinopathy Clinical Research Network compared PRP in 1 vs 4 sittings for severe nonproliferative diabetic retinopathy and PDR. ${ }^{15}$ The main finding was that PRP performed in a single sitting was not associated with any adverse effects on vision or macular thickness. However, the study was nonrandomized and MSPRP was undertaken during a long period of 12 weeks rather than in 2 to 3 biweekly sessions of 600 to 800 burns, which is common in the United Kingdom.

It is well reported by patients that conventional MSPRP is associated with discomfort, which in some cases can result in nonattendance for further treatment and poor outpatient follow-up. Though rare, patients who cannot tolerate the pain of PRP may require hospital admission, with subsequent indirect PRP undertaken in the operating room under local or general anesthesia.
The Pascal (OptiMedica, Santa Clara, California) photocoagulator (a pattern scanning laser) was introduced in 2005 for retinal photocoagulation. ${ }^{16}$ It partly automates the procedure using a brief pulse combined with rapid raster scan application of multiple spots, which allows shorter treatment delivery times. There may be less outer retina and retinal pigment epithelium damage due to less collateral thermal diffusion..$^{17} \mathrm{~A}$ recent study at our unit showed safe and well-tolerated application of Pascal laser photocoagulation. ${ }^{18}$

We conducted a prospective, randomized controlled trial to compare single-spot, 100-millisecond PRP across 3 sessions and multispot Pascal 20-millisecond PRP in a single session. The main hypothesis for the study was that Pascal 20-millisecond SS-PRP may not produce worsening of central macular thickness compared with conventional MS-PRP during treatment of PDR. The main aims of the study were to evaluate the effects of PRP on central subfield macular thickness and macular volume, and its secondary objectives were to look at the potential economic and functional benefits (field of vision, clinical efficacy, and treatment times) of SS-PRP.

\section{METHODS}

The Manchester Pascal Study protocol and informed consent forms were approved by the local research ethics committee. Data and safety monitoring was provided by an independent panel at both the University of Manchester and the research office at the Manchester Royal Eye Hospital. Patients who required PRP for newly diagnosed PDR were prospectively recruited. Forty eyes of 24 patients were studied between June 23, 2008, and July 10, 2009, and study visits included those 4 and 12 weeks posttreatment.

Following an updated refraction, baseline visual acuity (VA) was measured by a certified examiner using a logMAR vision chart at $2 \mathrm{~m}$ using the ETDRS method. The average of 2 ETDRS vision tests was used for analysis. All patients underwent slitlamp biomicroscopy, gonioscopy, and fundus fluorescein angiography at baseline. Two masked examiners at baseline graded the fundus fluorescein angiography images for entry into the study based on the study inclusion and exclusion criteria.

The major patient and study eye criteria are outlined in Table 1. The randomization sequence was generated using randomly permuted blocks, and a randomization table was created for the 40 eyes. After computerized randomization procedure, sequentially numbered, opaque, sealed envelopes were generated by the masked trial statistician. The treatmentallocation envelopes remained sealed until the interventions were assigned. Once a participant consented to the study, a research trial coordinator who was blinded to the treatment opened the sealed envelope and assigned participants to each group. The treating investigator and study participant were not blinded to treatment. Patients underwent either unilateral or bilateral PRP according to randomization treatment allocation. In cases of bilateral study eye eligibility, the right eye was randomized first with treatment allocation, followed by independent randomization and allocation of the left eye.

The primary efficacy end point was the mean change from baseline in Fourier-domain optical coherence tomography (FDOCT) central subfield retinal thickness (CRT) and macular volume at weeks 4 and 12. The main secondary efficacy end points were differences in mean change in VA over time and from baseline to weeks 4 and 12; the mean change in mean deviation (MD) of Swedish interactive threshold algorithm (SITA) visual field plots from baseline to weeks 4 and 12; the percentage of eyes that dem- 
onstrated a positive clinical effect from PDR regression over time and from baseline to week 12; and the mean treatment times of SS-PRP compared with MS-PRP. We used certified and blinded examiners to perform FD-OCT scans at each visit, and study participants underwent 2 visual field tests at each visit to exclude learning effects. Masked graders, who were blinded to the treatment arms of the study, undertook assessment of fundus photographs and fundus fluorescein angiography at baseline and at the final visit to grade the PDR activity postlaser. We used the mean scores of the 2 graders for statistical evaluation.

Safety end points included all adverse events reported spontaneously by study participants, elicited by investigators, and observed by investigators. Adverse events were graded as mild, moderate, or severe and were assessed as being either related or unrelated to the laser treatment. As part of ethical and good clinical practice, we recorded all serious adverse events whether or not they were deemed related to the treatment.

\section{FOURIER-DOMAIN OCT}

Baseline FD-OCT (Topcon 3D OCT-1000, Topcon Instruments, Newbury, England) was performed in the week before treatment. The scans were $6 \mathrm{~mm}$ in length, and each ETDRS macular grid was realigned with the anatomical fovea by a certified examiner. A certified and masked ophthalmic photographer at Manchester Royal Eye Hospital judged the analysis of mean changes in CRT. The macular volume within the central subfield was recorded using the Topcon software analysis of the ETDRS macular grid at the same anatomic point. The mean total macular volume was calculated using the sum of macular volume within the 9 ETDRS sectors.

\section{VISUAL FIELD TESTING}

The 24-2 SITA standard and Esterman binocular visual fields were recorded with a Humphrey 740 perimeter (Carl Zeiss Meditech Inc, Dublin, California) at baseline and 4 and 12 weeks postlaser. Two Humphrey visual field tests were undertaken for each study eye at every visit. The initial baseline visual field test was used as a training visual field to evaluate the patient's ability to competently undertake SITA visual field test and thus meet the inclusion criteria. We used the global index and MD to detect changes in the visual field. The MD is a measure of the average of differences from the normal expected value for a particular age. The mean value for MD was taken from the 2 SITA fields undertaken at the 4- and 12-week visits and compared with the second baseline visual field test. The Esterman plots were assessed according to the DVLA driving standards. The current minimum field of vision for safe driving is defined as a field of at least $120^{\circ}$ on the horizontal meridian measured using a target equivalent to the white Goldmann III4e settings. In addition, there should be no significant defect in the binocular field that encroaches within $20^{\circ}$ of fixation above or below the horizontal meridian (Figure 1). ${ }^{19}$

The Esterman results were examined by a member of both the Visual Standards Sub-Committee of the Royal College of Ophthalmologists and the Vision Panel for the DVLA. The examiner was blinded to the treatment allocations, and field tests were analyzed using the 2009 DVLA definition for the minimum field for safe driving (Figure 1). ${ }^{19}$

\section{PASCAL LASER PHOTOCOAGULATION SYSTEM}

The Pascal laser is a frequency-doubled, Nd:YAG, solid-state laser with a wavelength of $532 \mathrm{~nm}$. Photocoagulation is applied in a rapid pattern array with a pulse duration of 10 to 20 milliseconds. ${ }^{16}$ All treatment was performed with topical oxy-

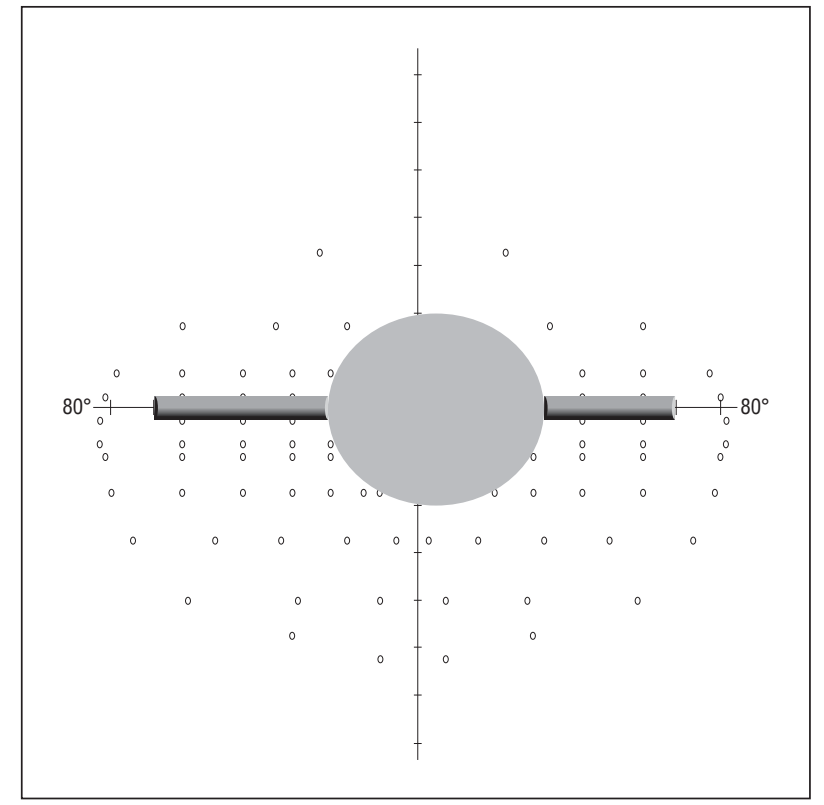

Figure 1. Current minimum field of vision for safe driving as defined by the Driving and Vehicle Licensing Authority (United Kingdom). The Esterman visual field is annotated to show at least $120^{\circ}$ on the horizontal meridian measured using a target equivalent to the white Goldmann III4e settings. In addition, there should be no significant defect in the binocular field that encroaches within $20^{\circ}$ of fixation above or below the horizontal meridian.

Table 2. Pascal Laser Parameters

\begin{tabular}{|c|c|c|}
\hline Parameter & $\begin{array}{c}\text { Single-Session } \\
\text { Group }\end{array}$ & $\begin{array}{l}\text { Multiple-Session } \\
\text { Group }\end{array}$ \\
\hline No. of sessions & 1: day 0 & $3:$ days 0,14 , and 28 \\
\hline No. of burns & 1500 & $\begin{array}{l}500 \text { per session; } \\
\text { total, } 1500\end{array}$ \\
\hline Type of laser & Pascal, 532 nm & Pascal, 532 nm \\
\hline Type of laser spot & $\begin{array}{l}\text { Pattern spot, } 5 \times 5 \text {, } \\
\quad 4 \times 4 \text { arrays }\end{array}$ & Single spot \\
\hline Spot size, $\mu \mathrm{m}$ & 400 & 400 \\
\hline Pulse duration, ms & 20 & 100 \\
\hline $\begin{array}{l}\text { Laser burn spacing, } \\
\text { burn-widths }\end{array}$ & 1.5 & 1.5 \\
\hline $\begin{array}{l}\text { Laser burn intensity, } \\
\text { ETDRS grade }\end{array}$ & $\begin{array}{l}2+, 3+; \text { mild } \\
\quad \text { gray-white }\end{array}$ & $\begin{array}{c}2+, 3+; \text { mild } \\
\text { gray-white }\end{array}$ \\
\hline
\end{tabular}

Abbreviation: ETDRS, Early Treatment Diabetic Retinopathy Study.

buprocaine hydrochloride, $0.4 \%$, administered to each patient. The laser parameters are presented in Table 2.

Twenty eyes underwent multispot, 20-millisecond SS-PRP using $5 \times 5$ and $4 \times 4$ multispot arrays, and 20 eyes underwent single-spot, 100-millisecond MS-PRP in 3 sessions across a period of 4 weeks. In both groups, threshold laser photocoagulation treatment was titrated to and designated by a mild graywhite burn (between grade $2+$ and $3+$ ) according to ETDRS guidelines. A single laser operator (M.M.K.M.) undertook the laser intervention for all eyes in the study. Before trial commencement, 2 experienced retinal specialists at Manchester Royal Eye Hospital externally validated the conventional single-spot and multispot Pascal laser techniques of the treating investigator (M.M.K.M.). Following a supervised 2-month period of laser training, the treating investigator was certified according to the study laser protocol and good clinical practice guidelines. During the study, threshold laser burn intensity was internally assessed and validated using 1-hour postlaser fundus photographs of laser PRP 


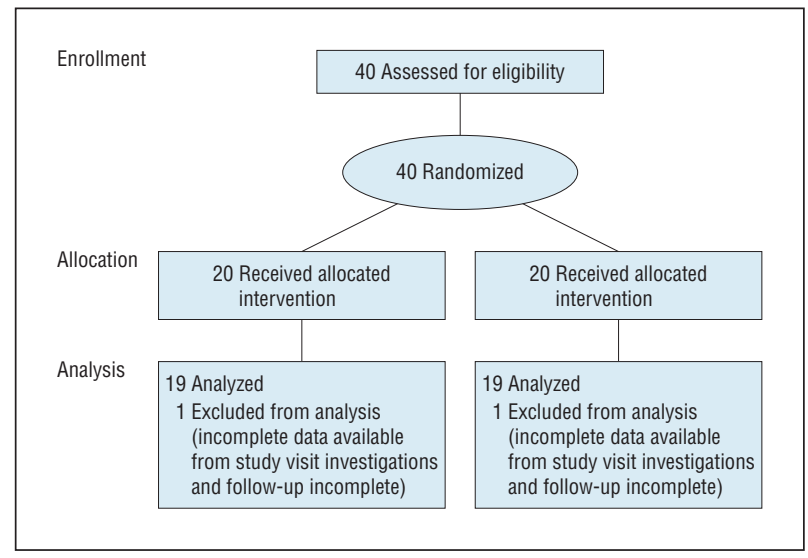

Figure 2. Flowchart for the study sample.

burns to ensure that threshold PRP laser burns were standardized for all eyes in both arms of the study.

One thousand five hundred photocoagulation burns comprised a full PRP treatment in both treatment groups, using a Mainster 165 PRP lens. Burn distribution for both groups were $1^{1 / 2}$ burn-widths apart, greater than 2 disc diameters temporal to the fovea, and no closer than 2 rows within the arcades, with burn placement as close to the ora serrata as possible.

Total treatment times were recorded by stopwatch from the initial titration burn until the final laser burn application for PRP in both groups. The timer was not stopped at any time during PRP treatments. The treatment time for MS-PRP was composed of the total times recorded for each of the three 500burn PRP sessions.

\section{ASSESSMENT OF CLINICAL EFFECT AND OUTCOMES}

Full 7-field standard ETDRS photographs were taken for color and red-free fundus photography at baseline. At both the 4and 12-week follow-up visits, 6-field ETDRS photographs were taken. Two masked retina specialists independently assessed the grade and activity of PDR at 12 weeks. The color and redfree grading photographs did not include any visible patterns of burns or cluster of single laser burns. These images captured the area of neovascularization and surrounding medium-sized vasculature only. The following 4-point visual grading scale was used by the graders to classify the activity of PDR neovascularization complexes after laser treatment: grade 3, complete regression of neovascularization; grade 2 , moderate regression of neovascularization with fibrosis; grade 1, mild regression of neovascularization with reduction of retinal vascular and neovascular caliber; and grade 0 , no change, or worsening of retinopathy and neovascularization. A positive clinical effect of PRP on PDR activity was designated for any grade from 1 to 3 in either group. A poor or inadequate response of PRP was designated by grade 0 . The decision to re-treat with further PRP was made according to standard ETDRS guidelines.

\section{STATISTICAL ANALYSIS}

We performed statistical analyses using Statistica, version 6 (StatSoft Inc, Tulsa, Oklahoma). We used the 2-tailed $t$ test to explore changes in CRT, macular volume, and visual field MD following treatment at specified points. The Fisher exact test was used to compare categorical outcomes. The null hypothesis was rejected for $P<.05$.

The sample size was based on the following assumptions: the test of significance should be 2-sided, with a significance
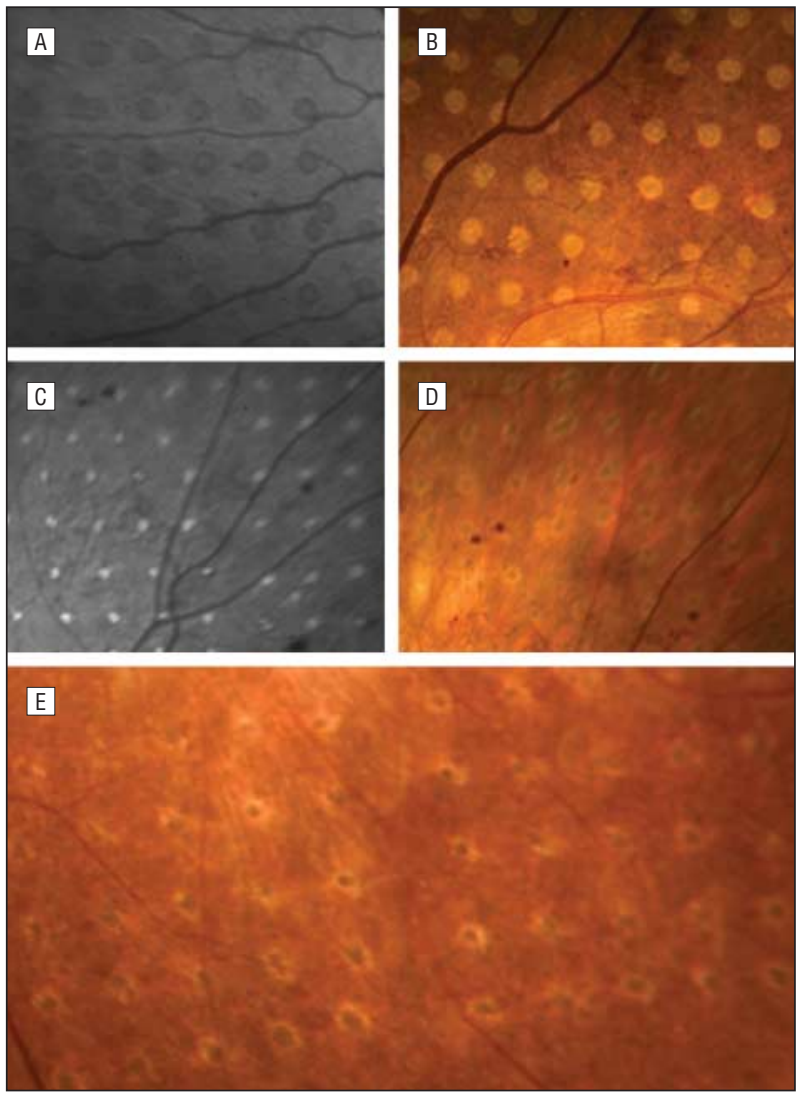

Figure 3. Pascal 20-millisecond single-session panretinal photocoagulation burns within $5 \times 5$ arrays shown at different times. A, Fundus autofluorescence photograph at 1 hour. B, Color fundus photograph at 1 hour. C, Fundus autofluorescence photograph at 4 weeks showing uniform increased autofluorescence within each pattern array. D, Color fundus photograph at 4 weeks showing localized laser burns. E, Color fundus photograph at 12 weeks showing partially pigmented laser burns within two $5 \times 5$ pattern arrays.

level of $P=.05$; the percentage of eyes in the MS-PRP group that develop a $25-\mu \mathrm{m}$ increase in CRT from baseline to week 12 was expected to be $41 \%$; and the percentage of eyes in the SS-PRP group that develop a $25-\mu \mathrm{m}$ increase in CRT from baseline to week 12 was expected to be $17 \%$; and considering previous studies, laser PRP effects on central retinal thickness within each subject group were normally distributed with an SD of 30. If the true difference in the experimental and control means for change in central retinal thickness after laser treatment is \pm 25 $\mu \mathrm{m}$, it was determined that a sample of at least 20 experimental subjects (SS-PRP) and 20 control subjects (MS-PRP) was required for an overall power of $80 \%$.

\section{RESULTS}

Forty eyes of 24 patients were studied between June 23, 2008, and July 10, 2009, and study visits included follow-up at 4 and 12 weeks posttreatment. The results from 1 eye from each treatment group were discarded because of incomplete data (Figure 2). The SS-PRP photocoagulation burns are shown in Figure 3. There was 1 eye from each treatment arm ( 1 of 20 in the SS-PRP group and 1 of 20 in the MS-PRP group). All of the 38 eyes, 19 in each arm, were analyzed. Sixteen patients underwent bilateral PRP, and 8 patients had unilateral PRP. The baseline characteristics are presented in Table 3 . 


\begin{tabular}{|c|c|c|c|c|c|}
\hline $\begin{array}{l}\text { Patient No./ } \\
\text { Sex/Age, y }\end{array}$ & $\begin{array}{c}\mathrm{HbA}_{1 \mathrm{c}} \\
\%\end{array}$ & $\begin{array}{c}\mathrm{BP}, \\
\mathrm{mm} \mathrm{Hg}\end{array}$ & $\begin{array}{c}\text { High } \\
\text { Cholesterol }\end{array}$ & Hypertension & $\begin{array}{l}\text { PDR Grade } \\
\text { at Baseline }\end{array}$ \\
\hline \multicolumn{6}{|c|}{ Single-Session Group } \\
\hline $1 / M / 29$ & 8.5 & $135 / 40$ & No & No & Moderate \\
\hline $2 / M / 48$ & 8.0 & $145 / 79$ & Yes & Yes & Moderate \\
\hline $3 / \mathrm{M} / 59$ & 7.5 & $139 / 40$ & No & Yes & Mild \\
\hline 4/M/37 & 10.0 & $145 / 87$ & No & No & Severe \\
\hline $5 / M / 37$ & 10.0 & $145 / 87$ & No & No & Severe \\
\hline 6/M/53 & 7.2 & $120 / 60$ & Yes & No & Moderate \\
\hline 7/M/53 & 7.2 & $120 / 60$ & Yes & No & Mild \\
\hline 8/F/34 & 7.0 & $135 / 75$ & Yes & Yes & Moderate \\
\hline $9 / M / 52$ & 5.5 & $158 / 90$ & Yes & Yes & Moderate \\
\hline $10 / F / 52$ & 7.0 & $137 / 80$ & Yes & No & Moderate \\
\hline $11 / \mathrm{M} / 51$ & 9.0 & $135 / 60$ & No & No & Severe \\
\hline $12 / \mathrm{M} / 30$ & 8.0 & $135 / 70$ & No & No & Moderate \\
\hline 13/M/34 & 8.0 & $130 / 60$ & No & No & Moderate \\
\hline $14 / F / 60$ & 8.0 & $150 / 80$ & No & Yes & Moderate \\
\hline 15/M/49 & 8.8 & $132 / 70$ & Yes & Yes & Moderate \\
\hline $16 / F / 48$ & 8.9 & $156 / 81$ & No & Yes & Moderate \\
\hline $17 / F / 52$ & 10.0 & $140 / 75$ & Yes & No & Moderate \\
\hline $18 / F / 48$ & 8.9 & $156 / 81$ & No & Yes & Mild \\
\hline 19/M/51 & 9.0 & $155 / 85$ & Yes & Yes & Moderate \\
\hline \multicolumn{6}{|c|}{ Multiple-Session Group } \\
\hline $1 / M / 29$ & 8.5 & $135 / 40$ & No & No & Moderate \\
\hline $2 / M / 42$ & 7.0 & $132 / 42$ & Yes & No & Moderate \\
\hline $3 / M / 42$ & 7.0 & $132 / 42$ & Yes & No & Moderate \\
\hline 4/M/52 & 6.3 & $118 / 68$ & No & Yes & Severe \\
\hline $5 / M / 52$ & 6.3 & $118 / 68$ & No & Yes & Mild \\
\hline 6/F/35 & 7.4 & $104 / 57$ & No & No & Mild \\
\hline 7/F/34 & 7.0 & $135 / 75$ & Yes & Yes & Mild \\
\hline 8/M/34 & 9.2 & $120 / 70$ & No & No & Moderate \\
\hline $9 / M / 34$ & 9.2 & $120 / 70$ & No & No & Mild \\
\hline 10/F/59 & 9.5 & $122 / 61$ & Yes & Yes & Moderate \\
\hline 11/M/52 & 5.5 & $158 / 90$ & Yes & Yes & Moderate \\
\hline 12/M/51 & 9.0 & $135 / 60$ & No & No & Severe \\
\hline $13 / F / 60$ & 8.0 & $150 / 80$ & No & Yes & Severe \\
\hline $14 / F / 44$ & 6.0 & $148 / 80$ & Yes & Yes & Moderate \\
\hline $15 / F / 44$ & 6.0 & $148 / 80$ & Yes & Yes & Moderate \\
\hline $16 / \mathrm{M} / 49$ & 8.8 & $132 / 70$ & Yes & Yes & Moderate \\
\hline $17 / F / 52$ & 10.0 & $140 / 75$ & Yes & No & Moderate \\
\hline 18/M/51 & 9.0 & $155 / 85$ & Yes & Yes & Severe \\
\hline 19/M/35 & 9.2 & $135 / 80$ & Yes & No & Mild \\
\hline
\end{tabular}

Abbreviations: BP, blood pressure; $\mathrm{HbA}_{1 \mathrm{c}}$, hemoglobin $\mathrm{A}_{1 \mathrm{c}}$. PDR, proliferative diabetic retinopathy.

Mean age in SS-PRP was 46 years (range, 29-60 years) and 44 years in MS-PRP (range, 29-60 years). The mean hemoglobin $\mathrm{A}_{1 \mathrm{c}}$ was $8.2 \%$ (SD, 1.2\%; range, 5.5\%-10\%) for the SS-PRP group, and 7.6\% (SD, 1.5\%; range, 5\%$10 \%$ ) in the MS-PRP group. There was no significant difference between baseline hemoglobin $\mathrm{A}_{1 \mathrm{c}}$ in the 2 groups $(P=.17)$. Complete data capture was obtained for 38 eyes at baseline and at the 4- and 12-week visits.

\section{LASER PARAMETERS}

All eyes received 1500 burns performed under topical anesthesia by a single investigator (M.M.K.M.). The mean power used for SS-PRP was significantly higher at $277 \mathrm{~mW}$ (range, 200-456 $\mathrm{mW}$; $P<.001$ ) than MS-PRP (143 mW; range, $104-188 \mathrm{~mW})$. The mean fluence for SS-PRP was $4.2 \mathrm{~J} / \mathrm{cm}^{2}$ (range, $3-7 \mathrm{~J} / \mathrm{cm}^{2}$ ), significantly lower than that for MS-PRP $\left(11.2 \mathrm{~J} / \mathrm{cm}^{2}\right.$; range, $\left.8-15 \mathrm{~J} / \mathrm{cm}^{2} ; P<.001\right)$.

The total PRP treatment time for 1500 burns was significantly shorter using multispot SS-PRP compared with single-spot MS-PRP (SS-PRP, mean, 5.04 minutes [SD,

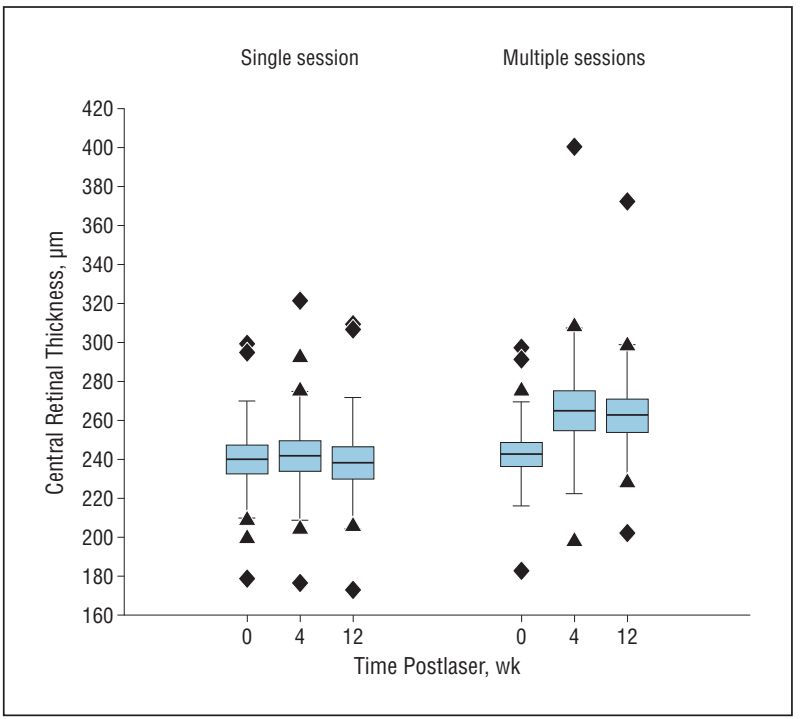

Figure 4. Central retinal thickness alterations over time. The horizontal line in the middle of each box indicates the mean, while the top and bottom borders of the boxes mark the standard error. The whiskers above and below the boxes mark the standard deviation. The triangles are outliers and the diamonds are extremes.

1.5 minutes]; vs MS-PRP, mean, 59.3 minutes [SD, 12.7 minutes]; $P<.001)$.

\section{GRADE OF RETINOPATHY}

We recruited patients with newly diagnosed PDR of any grade, with visible leakage from retinal neovascularization on fundus fluorescein angiography. The following grading scale was used at baseline: mild PDR, neovascularization elsewhere or at the disc that was less than the standard Airlie House photograph 10A; moderate PDR, neovascularization elsewhere greater than half a disc diameter and/or neovascularization at the disc greater than the standard Airlie House photograph 10A; and severe PDR, multiple neovascularizations elsewhere greater than half a disc diameters and/or forward neovascularization at the disc, preretinal hemorrhage, vitreous hemorrhage, or tractional retinal detachment.

In both the SS-PRP and MS-PRP groups, the mean and median severity of PDR was moderate. The types of retinopathy grade are shown in Table 3. Following treatment, there were no cases of clinically significant macular edema that required laser treatment in either group.

\section{MACULAR THICKNESS AND VOLUME}

The mean CRT at baseline was $240 \mu \mathrm{m}$ in the SS-PRP group (range, 178-299 $\mu \mathrm{m}$ ) and $242 \mu \mathrm{m}$ in the MS-PRP group (range, 182-297 $\mu \mathrm{m}$ ) as shown in Figure 4. In the SS-PRP group, there were no significant changes in mean CRT during followup. However, in the MS-PRP group, there were significant increases in mean CRT at 4 weeks and 12 weeks compared with baseline $(P<.001)$. After 4 weeks, the mean CRT increased by $2 \mu \mathrm{m}$ in the SS-PRP group compared with baseline (SD , $9.4 \mu \mathrm{m} ; 95 \%$ confidence interval [CI],-6.37 to 2.68 ; $P=.4$ ) and increased significantly by $22 \mu \mathrm{m}$ (SD, $21.6 \mu \mathrm{m}$; $95 \% \mathrm{CI},-32.25$ to $-10.75 ; \mathrm{P}<.001)$ in the MS-PRP group

(REPRINTED) ARCH OPHTHALMOL/VOL 128 (NO. 5), MAY $2010 \quad$ WWW.ARCHOPHTHALMOL.COM 


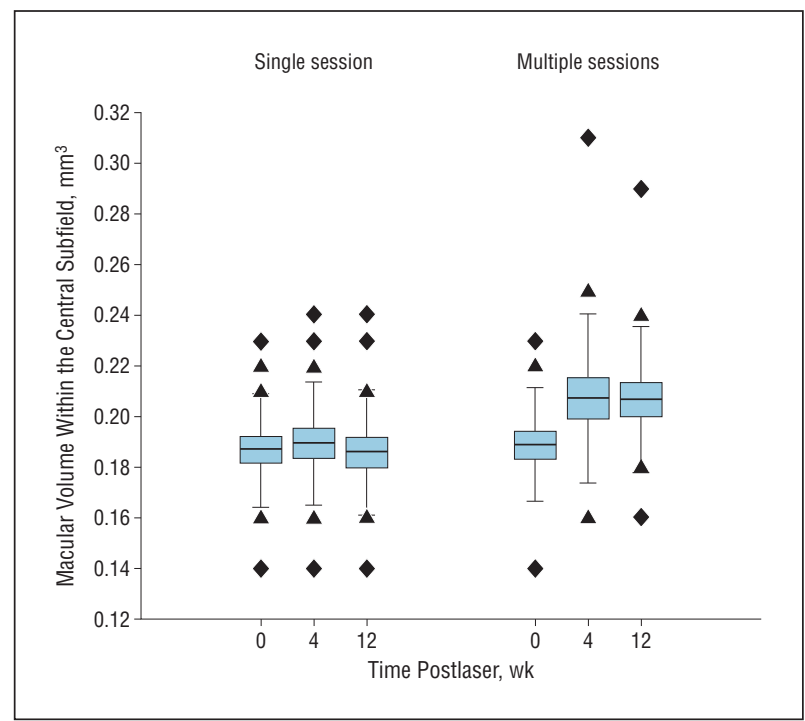

Figure 5. Macular volume changes within the central subfield over time. The horizontal line in the middle of each box indicates the mean, while the top and bottom borders of the boxes mark the standard error. The whiskers above and below the boxes mark the standard deviation. The triangles are outliers and the diamonds are extremes.

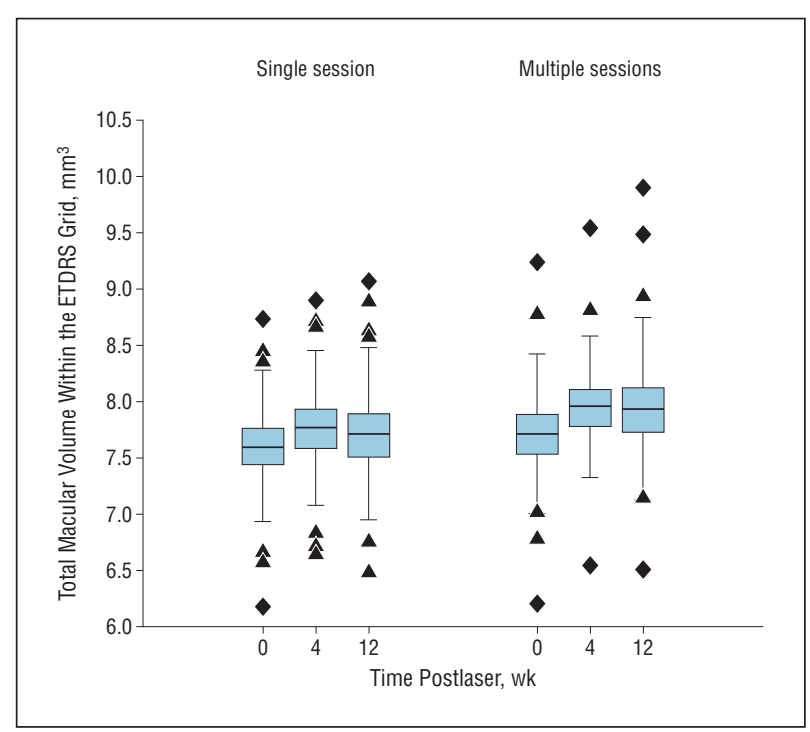

Figure 6. Total macular volume changes over time. The horizontal line in the middle of each box indicates the mean, while the top and bottom borders of the boxes mark the standard error. The whiskers above and below the boxes mark the standard deviation. The triangles are outliers and the diamonds are extremes. ETDRS indicates Early Treatment Diabetic Retinopathy Study.

from baseline. At the final, 12-week visit, mean CRT had decreased by $2 \mu \mathrm{m}$ in the SS-PRP group from baseline (SD, 9.5 $\mu \mathrm{m} ; 95 \% \mathrm{CI},-2.86$ to 6.33$)$. At 12 weeks following MS-PRP, mean change in CRT remained $20 \mu \mathrm{m}$ greater than baseline (SD, $18.6 \mu \mathrm{m} ; 95 \% \mathrm{CI},-28.75$ to $-10.82 ; P<.001$ ).

The mean macular volume within the central subfield at baseline was $0.18 \mathrm{~mm}^{3}$ in the SS-PRP group and 0.19 $\mathrm{mm}^{3}$ in the MS-PRP group (Figure 5). In the SS-PRP group, there were no significant changes in mean macular volume during follow-up; however, in the MS-PRP group, there was a significant increase in macular volume $(P<.001)$.

Mean total macular volume was $7.65 \mathrm{~mm}^{3}$ in the SSPRP group and $7.72 \mathrm{~mm}^{3}$ in the MS-PRP group at base-

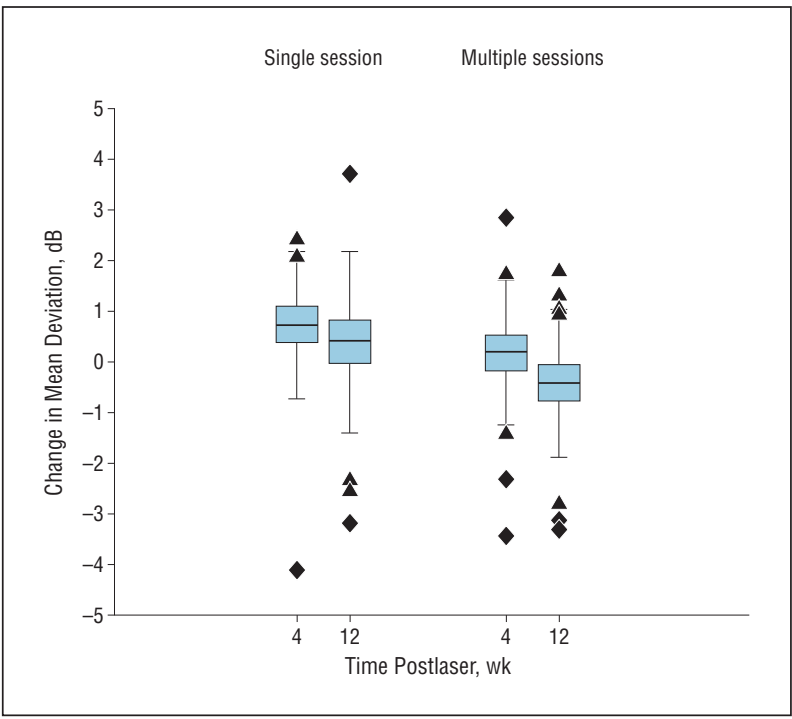

Figure 7. Change in mean deviation of visual field plots over time. The horizontal line in the middle of each box indicates the mean, while the top and bottom borders of the boxes mark the standard error. The whiskers above and below the boxes mark the standard deviation. The triangles are outliers and the diamonds are extremes.

line (Figure 6). In the SS-PRP group, there were no significant changes in the total macular volume during follow-up; however, in the MS-PRP group, there was a significant increase at both 4 weeks $(P=.004)$ and 12 weeks $(P=.01)$.

\section{CLINICAL EFFECTS OF LASER}

There were no significant differences between the results of the 2 masked graders for PRP outcomes (SS-PRP, P > .99; MS-PRP, $P=.75$ ). For the SS-PRP, a positive effect was observed in $74 \%$ of eyes following treatment ( 3 of 19 at grade 3,1 of 19 at grade 2,9 of 19 at grade 1 , and 6 of 19 at grade 0 ). A positive clinical effect was observed in $53 \%$ of eyes in the MS-PRP group postlaser ( 1 of 19 at grade 3, 2 of 19 at grade 2, 7 of 19 at grade 1 , and 9 of 19 at grade 0 ). Additional PRP was performed in all eyes with persistent, active PDR at the 12-week visit. There were no significant differences between the effects of laser on PDR activity between SS-PRP and MS-PRP $(P=.31)$. There was no association between either prelaser hypertension or hypercholesterolemia status and clinical response to PRP in both groups.

\section{VISUAL FIELD}

The average MD at baseline was $-5.94 \mathrm{~dB}(\mathrm{SD}, 2.9 \mathrm{~dB})$ in the SS-PRP group and $-4.62(\mathrm{SD}, 3.5 \mathrm{~dB} ; P=.29)$ in the MSPRP group. During follow-up in the SS-PRP group, the MD increased by $0.73 \mathrm{~dB}(P<.05)$ at 4 weeks and was $0.40 \mathrm{~dB}$ greater at 12 weeks $(P=.36$; both changes compared with baseline). In the MS-PRP group, there was a 0.39-dB increase in MD at 4 weeks from baseline $(P=.58)$ and a smaller, 0.42 $\mathrm{dB}$ decrease at 12 weeks compared with baseline $(P=.24)$.

Comparing the MD changes between both arms of the study, the average MD increased by $0.54 \mathrm{~dB}$ after SSPRP compared with MS-PRP at 4 weeks $(P=.18)$. There was a further increase of $0.81 \mathrm{~dB}(P=.12)$ in MD after 12 weeks between SS-PRP and MS-PRP (Figure 7). 
The Esterman fields were assessed in a masked, randomized method according to the DVLA driving standards. A single patient in the SS-PRP group failed the driving visual field test prelaser and this did not change at the 3-month visit postlaser. A single patient failed the driving visual field test following MS-PRP.

\section{VISUAL ACUITY}

The baseline ETDRS VA was 77 letters (SD, 9.9 letters) in the SS-PRP group and 79 letters (SD, 7.9 letters; $P=.57$ ) in the MS-PRP group. After 4 weeks, there was a 2-letter increase (SD, 9.3 letters) in VA in the SS-PRP group and a 1-letter increase (SD, 18.9 letters) in the MS-PRP group. In the SS-PRP group at 12 weeks, the VA had increased 4 letters (SD, 6 letters) from baseline compared with MS-PRP $(P=.25)$. There was no obvious correlation between VA changes and retinal thickness alterations between the groups at any point.

\section{COMPLICATIONS}

There were no immediate or short-term ocular complications following either SS-PRP or MS-PRP. There were no reported adverse or serious adverse events. Intraocular pressure was stable in all eyes throughout the study. No cases of vitreous hemorrhage or serious adverse events were recorded for any study eye. In the Pascal multispot SS-PRP group, there were no signs of intraretinal hemorrhage or blood vessel compromise at the locations of photocoagulation burns.

\section{COMMENT}

The Manchester Pascal Study prospectively investigated patients with newly diagnosed PDR who were without clinically significant macular edema or any other associated ocular morbidity. Pascal laser treatment was randomly allocated between either MS-PRP using a longpulse (100-millisecond) and single-spot mode or Pascal laser SS-PRP using a medium-pulse (20-millisecond) and multispot pattern scanning mode. The CRT was not significantly affected following SS-PRP over time. However, there was an initial 22- $\mu \mathrm{m}$ increase in CRT after MSPRP, followed by persistent and significant central retinal thickening of $20 \mu \mathrm{m}$ at 12 weeks.

Laser photocoagulation has been shown to produce both a thermal rise and thermal spread within the outer retina. Intraretinal inflammation following PRP has been associated with macular edema and visual loss. ${ }^{7,20}$ A variety of theories for PRP-induced inflammation exist. These include leukocyte-endothelial cell interactions that lead to inflammatory maculopathy after PRP in animal models and correlation of laser-induced increased cytokine release with retinal capillary hyperpermeability. ${ }^{21,22}$ Shimura et $\mathrm{al}^{23}$ reported transient increases in macular thickness after MS-PRP using a krypton red laser (2000 burns, 200- to 500- $\mu \mathrm{m}$ spot size, 150- to 200millisecond pulse).

It is important to appreciate the relationship of laser power to fluence for contemporary treatment of PDR. Flu- ence is calculated as (power $\times$ time)/area. Our series has demonstrated that the fluence required to produce a threshold $2+$ and $3+$ ETDRS grade burn on the retina was significantly lower for a 20-millisecond compared with a 100-millisecond pulse. We did not observe any detrimental effects on clinical efficacy using 20 milliseconds for SS-PRP compared with 100 milliseconds in MSPRP at different levels of fluence.

We hypothesized that in the treatment of PDR, the severity of visual field loss may correlate with burn density, longer pulse, and higher fluence. To reduce the risk of complications after PRP, we determined that the optimal regimen might be to use a scatter PRP technique of uniform treatment burns over a wider retinal area up to the ora serrata. The Pascal system allows each pattern array to have burns uniformly placed, and we used arrays with 1.5spot spacing for the SS-PRP group. The ETDRS recommended 1-burn width spot spacing; however, we aimed to maximize retinal laser coverage in a single sitting and to allow potential re-treatments to be safely placed between previous arrays of burns, thus preventing overlapping laser burns and nerve fiber layer defects. ${ }^{2}$

Visual field scores were favorable for both groups, with no worsening of visual field MD observed after SS-PRP. Once again, owing to the small sample size, the improvements in visual field after PRP should be viewed tentatively. In contrast to our study, the ETDRS reported significant reductions in MD scores after full-scatter PRP. ${ }^{8}$ Pahor $^{12}$ used a similar PRP strategy to ours (1505 burns, 500- $\mu \mathrm{m}$ spot size, 100-millisecond pulse, 200- to 300-mW power) and found reductions in central visual field scores and MD after both mild- and full-scatter PRP. Similar findings have been reported by Henricsson and Heijl, ${ }^{11}$ who used parameters similar to those in our MS-PRP group; however, a half-burn spot spacing was preferred in their study.

In the 1990s, there were a number of studies that investigated PRP and whether its effect on the visual field meant that patients no longer met the standard needed to drive in the UK. ${ }^{24}$ Fifty percent failed the DVLA driving standard using treatment ranging from 164 to 5917 photocoagulation burns. ${ }^{13}$ Hulbert and Vernon ${ }^{25}$ have reported results of PRP using different pulse durations on the visual field. In our study, 16 patients underwent bilateral PRP with SS-PRP and/or MS-PRP. One patient had a preexisting failure prelaser in the SS-PRP group, which did not alter postlaser. No eyes had new driving test failures following 20-millisecond SS-PRP treatment. A single patient ( $6 \%$ of total eyes that underwent bilateral PRP) in the MS-PRP group failed the driving field test at 3 months as a consequence of laser treatment. For all eyes in the study, the rate of driving visual field test failure was $2.7 \%$ post-PRP.

The purpose of PRP in PDR is to prevent moderate to severe visual loss. Vision after MS-PRP remained stable during follow-up. Eyes in the SS-PRP group had no worsening in VA compared with the MS-PRP group at 12 weeks. We were unable to find any correlation between VA and retinal thickness changes in either group.

Until now, Doft and Blankenship ${ }^{14}$ have reported on the only RCT comparing SS-PRP with MS-PRP in 50 eyes. They used fewer photocoagulation burns than our study and their laser parameters were a spot size of $500 \mu \mathrm{m}$ and a pulse of 
100 milliseconds. A number of significant yet transient complications occurred, including exudative retinal detachment, choroidal detachment, raised intraocular pressure, angle closure, and macular edema. We did not encounter any clinical signs of any of these complications in any patient during our study. Furthermore, no differences between SS-PRP and MS-PRP were observed with relation to visual field scores and clinical outcomes.

The recent Diabetic Retinopathy Clinical Research Network study used similar entry criteria as our study $.{ }^{15} \mathrm{Mul}-$ tiple laser operators were involved, with PRP parameters ranging from 1260 to 1274 burns, 50- to 200-millisecond pulse duration, 200- to 500- $\mu \mathrm{m}$ spot size, and 250- to 280-mW laser power. Within the treatment groups, 46 eyes with PDR underwent PRP in 1 sitting and 34 eyes with PDR were treated across 4 sittings. Similar laser powers were used in both groups and there was a greater increase in macular thickness observed at 4 weeks in the single-sitting group compared with the 4-sittings group. Our results suggest fewer adverse effects from SS-PRP than MS-PRP in relation to macular thickness, total macular volume, and visual field scores up to 12 weeks. An important issue for patients undergoing PRP is whether the driving visual field may be adversely affected by bilateral PRP. The Diabetic Retinopathy Clinical Research Network study did not include data on whether or not patients continued to meet the standard needed to drive after PRP.

The prelaser hemoglobin $A_{1 c}$ levels, grade of baseline PDR, and mean blood pressure levels were equivalent in both groups. At the time of conception, a safe number of PRP burns using the Pascal system was unknown and 1500 burns was selected according to ETDRS guidelines for conventional treatment. We found a $74 \%$ positive clinical effect after 20-millisecond, multispot SSPRP compared with 53\% after single-spot MS-PRP. However, the sample size is too small and our follow-up is too short to draw definite conclusions regarding differences in clinical efficacy between the 2 groups.

The Pascal photocoagulator system of treating diabetic patients with multispot PRP may have a greater implication in terms of hospital or office diabetic eye care. A full SS-PRP treatment may be delivered using 20millisecond multispot burns with a significant reduction in treatment time and equivalent clinical outcomes compared with conventional pulse, single-spot MSPRP. Multiple-session PRP may be undertaken in 2 to 4 sessions during 4 to 8 weeks in the UK or United States. ${ }^{15}$ The main advantage is the saving of time for both the patient and the physician using a SS-PRP strategy. Furthermore, SS-PRP treatment permits an earlier clinical reevaluation post-PRP, since an additional 4- to 8-week delay is incurred with an MS-PRP approach before PDR responses to PRP may be re-examined.

A simple cost-minimization analysis may be applied to the PRP strategies, as the clinical outcomes are equivalent. Input costs for a hospital laser clinic may be reduced with an SS-PRP approach compared with biweekly MS-PRP treatments. More patients may potentially be treated using multispot SS-PRP per clinic session with associated shorter waiting times, both for a laser appointment as well as in the waiting room on the treatment day. The cumulative cost savings for the hospital eye service is clearly visible. However, the prices of new systems such as the Pascal photocoagulator need to be accounted for in such cost analysis.

Currently, the Diabetic National Service Framework (UK) and the English National Screening Programme (UK) have recommended a 2-week target for the primary laser treatment of newly diagnosed PDR. In current practice using conventional argon green laser in MSPRP, this target may be only achievable in around $75 \%$ of patients. A potential target of $95 \%$ patient treatment may be realistic as a consequence of the previously discussed savings in treatment times with increased flow of patients through treatment clinics.

The main limitation of our study is the small sample size and short follow-up time. The main aims of this study were to evaluate the safety profile of multispot SS-PRP compared with conventional single-spot MS-PRP, and potential adverse effects on macular thickness and the visual field. We have been able to fully investigate these important safety issues regarding the application of multispot SS-PRP and are able to draw significant conclusions regarding these primary outcomes using this sample size. This trial has produced useful laser parameters relevant for clinical practice and demonstrated functional effects and similar clinical efficacy between both types of laser regimen.

The strengths of our study include external validation of the PDR grade and clinical outcomes of the treatment by masked graders and the use of certified and masked analysis of FD-OCT scan data for CRT and macular volume. Furthermore, an independent evaluator assessed driving visual fields according to DVLA. The positive results of our study would merit further evaluation of multispot, 20-millisecond SS-PRP compared with 100millisecond, single-spot MS-PRP in a larger multicenter trial.

Although our trial was intended to assess the noninferiority of SS-PRP compared with MS-PRP, the results of our study suggest that multispot SS-PRP may be more safely and rapidly performed with 1500 burns compared with conventional MS-PRP with similar clinical effects on PDR clinical outcomes. There was no detrimental effect on driving visual fields after SS-PRP. The use of medium pulse duration requires less fluence, and this may prevent either retinal thickening or visual field deterioration compared with 100-millisecond, single-spot MS-PRP.

Importantly, multispot SS-PRP may increase patient compliance with laser treatment and reduce the number of hospital visits that may incur additional emotional and financial costs for the patient. These cost savings may reduce the increasing financial burden and waiting times for diabetic eye care services within the hospital over the long term.

Submitted for Publication: October 14, 2009; final revision received December 7, 2009; accepted December 24, 2009.

Correspondence: Paulo E. Stanga, MD, University of Manchester, Manchester Royal Eye Hospital, Oxford Road, Manchester M139WH, England (retinaspecialist @btinternet.com). 
Author Contributions: Dr Muqit had full access to all of the data in this article and takes responsibility for the integrity of the data and the accuracy of the data analysis. Financial Disclosure: Dr Marcellino is an employee of OptiMedica Corporation. Dr Stanga has received financial support from OptiMedica Corporation.

Funding/Support: This study was supported by OptiMedica Corporation, the manufacturer of the Pascal laser; the Manchester Academic Health Sciences Centre; and the National Institute for Health Research Manchester Biomedical Research Centre.

Previous Presentations: This work was presented at the Annual Meeting of the Association of Research and Vision in Ophthalmology, Ft Lauderdale, Florida, May 3-7, 2009; at the Royal College of Ophthalmologists Annual Congress, Birmingham, England, May 19-21, 2009; and at the joint American Academy of Ophthalmology and Pan-American Association of Ophthalmology Annual Meeting, San Francisco, California, October 24-27, 2009. Additional Contributions: Binu John, FRCOphth, and Rod MacKenzie, FRCOphth, Manchester Royal Eye Hospital, assisted in the grading of clinical photographs. Faruque D. Ghanchi, FRCOphth, assisted in grading driving visual fields. We are grateful to all staff within the Clinical Imaging Unit at Manchester Royal Eye Hospital for performing OCT and fundus photography.

\section{REFERENCES}

1. The Diabetic Retinopathy Study Research Group. Preliminary report on effects of photocoagulation therapy. Am J Ophthalmol. 1976;81(4):383-396.

2. Early Treatment of Diabetic Retinopathy Study Research Group. Techniques for scatter and local photocoagulation treatment of diabetic retinopathy: Early Treatment of Diabetic Retinopathy Study Report No. 3. Int Ophthalmol Clin. 1987; 27(4):254-264.

3. Stanga PE, Reck AC, Hamilton AM. Micropulse laser in the treatment of diabetic macular oedema. Semin Ophthalmol. 1999;14(4):210-213.

4. Framme C, Schuele G, Roider J, Birngruber R, Brinkmann R. Influence of pulse duration and pulse number in selective RPE laser treatment. Lasers Surg Med. 2004:34(3):206-215.

5. Toth CA, Narayan DG, Cain CP, et al. Pathology of macular lesions from subnanosecond pulses of visible laser energy. Invest Ophthalmol Vis Sci. 1997; 38(11):2204-2213

6. Zweng HC, Little HL, Hammond AH. Complications of argon laser photocoagulation. Trans Am Acad Ophthalmol Otolaryngol. 1974;78(2):OP195-0P204.

7. McDonald HR, Schatz H. Visual loss following panretinal photocoagulation for proliferative diabetic retinopathy. Ophthalmology. 1985;92(3):388-393.
8. Early Treatment of Diabetic Retinopathy Study Research Group. Early photocoagulation for diabetic retinopathy: ETDRS report number 9. Ophthalmology. 1991; 98(5)(suppl):766-785.

9. Paulus YM, Jain A, Gariano RF, et al. Healing of retinal photocoagulation lesions. Invest Ophthalmol Vis Sci. 2008;49(12):5540-5545.

10. Maeshima K, Utsugi-Sutoh N, Otani T, Kishi S. Progressive enlargement of scattered photocoagulation scars in diabetic retinopathy. Retina. 2004;24(4):507511.

11. Henricsson M, Heijl A. The effect of panretinal laser photocoagulation on visual acuity, visual fields and on subjective visual impairment in preproliferative and early proliferative diabetic retinopathy. Acta Ophthalmol (Copenh). 1994;72 (5):570-575.

12. Pahor D. Visual field loss after argon laser panretinal photocoagulation in diabetic retinopathy: full- versus mild-scatter coagulation. Int Ophthalmol. 1998; 22(5):313-319.

13. Pearson AR, Tanner V, Keightley SJ, Casswell AG. What effect does laser photocoagulation have on driving visual fields in diabetics? Eye (Lond). 1998;12 (pt 1):64-68.

14. Doft BH, Blankenship GW. Single versus multiple treatment sessions of argon laser panretinal photocoagulation for proliferative diabetic retinopathy. Ophthalmology. 1982;89(7):772-779.

15. Brucker AJ, Qin H, Antoszyk AN, et al; Diabetic Retinopathy Clinical Research Network. Observational study of the development of diabetic macular edema following panretinal (scatter) photocoagulation given in 1 or 4 sittings. Arch Ophthalmol. 2009;127(2):132-140.

16. Blumenkranz MS, Yellachich D, Andersen DE, et al. Semiautomated patterned scanning laser for retinal photocoagulation. Retina. 2006;26(3):370-376.

17. Jain A, Blumenkranz MS, Paulus $Y$, et al. Effect of pulse duration on size and character of the lesion in retinal photocoagulation. Arch Ophthalmol. 2008; 126(1):78-85.

18. Sanghvi C, McLauchlan R, Delgado C, et al. Initial experience with the Pascal ${ }^{\text {" }}$ photocoagulator: a pilot study of 75 procedures. Br J Ophthalmol. 2008;92 (8):1061-1064.

19. Driver and Vehicle Licensing Agency. For Medical Practitioners: At a Glance Guide to the Current Medical Standards of Fitness to Drive. Swansea, Wales; Drivers Medical Group, DVLA: February 2009. http://www.dvla.gov.uk/medical/ataglance .aspx. Accessed September 7, 2009.

20. McDonald HR, Schatz H. Macular edema following panretinal photocoagulation. Retina. 1985;5(1):5-10.

21. Nonaka A, Kiryu J, Tsujikawa A, et al. Inflammatory response after scatter laser photocoagulation in nonphotocoagulated retina. Invest Ophthalmol Vis Sci. 2002 43(4):1204-1209.

22. Dharma S, Bazan HE, Peyman GA, Atef MS. Production of platelet-activating factor in photocoagulated retinas. Curr Eye Res. 1991;10(11):1031-1035.

23. Shimura M, Yasuda K, Nakazawa T, Kano T, Ohta S, Tamai M. Quantifying alterations of macular thickness before and after panretinal photocoagulation in patients with severe diabetic retinopathy and good vision. Ophthalmology. 2003; 110(12):2386-2394.

24. Buckley SA, Jenkins L, Benjamin L. Fields, DVLC and panretinal photocoagulation. Eye (Lond). 1992;6(pt 6):623-625.

25. Hulbert MFG, Vernon SA. Passing the DVLC field regulations following bilateral pan-retinal photocoagulation in diabetics. Eye (Lond). 1992;6(pt 5):456-460. 